\title{
MIMO-OFDM SYSTEMS WITH MULTI-USER INTERFERENCE
}

\author{
Masoud Olfat, Farrokh R. Farrokhi, Mehdi Alasti, and K. J. Ray Liu
}

Electrical and Computer Eng. Department, University of Maryland, College Park, MD 20742

\begin{abstract}
In this paper we consider downlink transmission in a multiuser environment, where each base station transmits multiple data streams to its corresponding mobiles using Multiple Input Multiple Output (MIMO) OFDM. Using distributed schemes, we find the optimal transmit and receive weight vectors, to maximize total data rate for each user. We consider two scenarios, where in one the total power per user and in the other total power per subcarrier is fixed. In each case, we propose iterative algorithms to maximize the allocated rate per user. We will discuss the convergence of our schemes and compare their performances with that of the previous works.
\end{abstract}

\section{INTRODUCTION}

It has been shown that using multiple antenna transmission in wireless communication systems results in significant capacity improvement without the need to allocate extra power or bandwidth [1], [2]. Multiple antennas are used to achieve beamforming gain , spatial diversity by using space-time codes, or spatial multiplexing [1]. In spatial multiplexing, signal processing is used at both the transmitter and receiver to decompose multiple streams transmitted from the transmitter [3]. In spatial multiplexing, the knowledge of channel condition is optimally performed, assuming that the channel is known at the transmitter as well as in the receiver [2]. Some researchers have designed tranmit schemes to maximize the information theoretic sum capacity of multiple access channels [4].

Different subcarriers in an Orthogonal Frequency Division Multiplexing (OFDM) system experience different attenuations. The exploitation of multiple transmit and receive antennas MIMO OFDM both in the form of beamforming and space-frequency coding [5] has recently found much attention. Many of these schemes have considered a single transmitter and receiver. However, in addition to the effect of multipath fading, in a multi-user environment, the interference from other transmitters, plays a detrimental role in degrading the system performance.

In [6], we proposed joint beamforming and power control for multiuser OFDM systems. In this paper, we will consider using MIMO-OFDM systems in a multiuser environment, where multiple data streams are transmitted form each transmitter. Unlike previous works in this context, we will consider a multi-cell system, where overall transmit power is assumed to be fixed for each user. We will increase each user's data rate by adjusting transmit and receive weight vectors according to an iterative water-filling algorithm. The convergence of each scheme is also discussed.

The rest of this paper is organized as follows: Section 2 describes the system model. Section 3 quantifies the overall OFDM data rate and the optimization of the transmit and receive weight vectors. We also provide iterative algorithms to maximize each user data rate. In Section 4 we will evaluate the performance of the proposed algorithms and finally, Section 5 concludes the paper.

\section{SYSTEM MODEL}

We consider a downlink multi-user OFDM system consisting of $M$ cells, one base station equipped with $N_{t}$ transmit antennas per cell, and $N_{m}$ mobiles equipped with $N_{r}$ receive antennas in the $m^{t h}$ cell. Total number of mobiles is considered to be $N$. That is, there are $N$ co-channel link pairs for uplink and downlink transmission. A similar analysis can be applied to uplink transmission. $T$ independent data streams are transmitted from each base station, where a different transmit weight vector is calculated for transmitting each stream to the corresponding mobiles. Assuming that the number of OFDM subcarriers, $K$, is large enough, each subchannel is assumed to follow a flat fading quasi static model, where the subchannel is constant over an OFDM blocks. The frequency domain subchannel link gains from base station $m$ to mobile $n$ at frequency carrier $k$ is denoted by an $N_{r}$ by $N_{t}$ matrix $\mathbf{H}_{m n}^{k}$, whose $(i, j)$-th element $\left[\mathbf{H}_{m n}^{k}\right]_{i j}$, represents the channel link gain from the transmit antenna $j$ to receive antenna $i$. The received signal at the $k^{t h}$ carrier of the $i^{t h}$ mobile is given by

$$
\mathbf{x}_{i}^{k}=\mathbf{W}_{i}^{k}{ }^{H}\left(\sum_{m=1}^{M} \mathbf{H}_{m i}^{k} \mathbf{V}_{m}^{k} \mathbf{s}_{m}^{k}+\mathbf{n}_{i}^{k}\right)
$$


where $\mathbf{s}_{m}$ is the data vector of size $T$ that is intended to be transmitted to user $m$, and its covariance $\Phi_{m}^{k}$ is defined by $\Phi_{m}^{k}=E\left\{\mathbf{s}_{m}^{k}\left(\mathbf{s}_{m}^{k}\right)^{H}\right\}$. The thermal noise vector at the $i^{\text {th }}$ receiver, $\mathbf{n}_{i}^{k}$ is of size $N_{r}$, whose spatial covariance matrix is diagonal with equal power $\left(\sigma_{i}^{k}\right)^{2}$ per antenna. The $N_{r} \times T$ matrix $\mathbf{W}_{i}^{k}$ and $N_{t} \times T$ matrix $\mathbf{V}_{m}^{k}$ represent the receive and transmit weight vectors, respectively. Each column of these matrices corresponds to one of the streams. Total transmit power at the $k^{t h}$ subcarrier is $\operatorname{tr}\left(\mathbf{V}_{m}^{k}{ }^{H} \Phi_{m}^{k} \mathbf{V}_{m}^{k}\right)$. Assuming that the transmitted streams from different users are independent of each other and also independent of noise samples, the total covariance matrix at receiver $i$ is given by

$$
\mathbf{X}_{i}^{k}=\mathbf{W}_{i}^{k^{H}} \mathbf{H}_{i i}^{k} \mathbf{V}_{i}^{k} \Phi_{i}^{k} \mathbf{V}_{i}^{k}{ }^{H} \mathbf{H}_{i i}^{k}{ }^{H} \mathbf{W}_{i}^{k}+\mathbf{W}_{i}^{k}{ }^{H} \mathbf{Q}_{i}^{k} \mathbf{W}_{i}^{k},
$$

where

$$
\mathbf{Q}_{i}^{k}=\sum_{m \neq i} \mathbf{H}_{m i}^{k} \mathbf{V}_{m}^{k} \Phi_{m}^{k} \mathbf{V}_{m}^{k}{ }^{H} \mathbf{H}_{m i}^{k}{ }^{H}+\sigma_{i}^{k^{2}} \mathbf{I}_{N_{r}},
$$

is the covariance of the interference and noise at the $k^{\text {th }}$ subcarrier of the $i^{\text {th }}$ receiver.

\section{MAXIMUM THROUGHPUT POLICY}

If the transmit signal is assumed to be Gaussian or the number of users is large enough (using central limit theorem in this case), we can assume a Gaussian distribution for the interference. Furthermore, we follow two scenarios. In the first one, we assume a fix transmit power policy per subcarrier where every user adapts its data rate with the total transmit power across its antennas held constant. In the second scheme, the transmit power is fixed per user. For notation simplicity, we will drop the index $k$. The mutual information at subcarrier $k$ can be expressed as:

$$
\begin{aligned}
\mathcal{I}_{i} & =\log _{2}\left|\mathbf{W}_{i}{ }^{H}\left(\mathbf{H}_{i i} \mathbf{V}_{i} \Phi_{i} \mathbf{V}_{i}{ }^{H} \mathbf{H}_{i i}{ }^{H}+\mathbf{Q}_{i}\right) \mathbf{W}_{i}\right| \\
& -\log _{2}\left|\mathbf{W}_{i}{ }^{H} \mathbf{Q}_{i} \mathbf{W}_{i}\right|
\end{aligned}
$$

We would like to maximize $\mathcal{I}_{i}$, with the constraint $\operatorname{tr}\left(\mathbf{V}_{m}^{k}{ }^{H} \Phi_{m}^{k} \mathbf{V}_{m}^{k}\right) \leq P_{m}^{k}$. Using the relation $\operatorname{det}(I+$ $A B)=\operatorname{det}(I+B A)$, we can change the mutual information to

$$
\begin{aligned}
\mathcal{I}_{i}= & \log _{2} \mid \mathbf{H}_{i i}^{H} \mathbf{W}_{i}\left(\mathbf{W}_{i}{ }^{H} \mathbf{Q}_{i} \mathbf{W}_{i}\right)^{-1} \\
& \times \mathbf{W}_{i}{ }^{H} \mathbf{H}_{i i} \mathbf{V}_{i} \Phi_{i} \mathbf{V}_{i}^{H}+I_{N_{t}} \mid .
\end{aligned}
$$

The MMSE receiver weight vectors are given by

$$
\mathbf{W}_{i}=\mu \mathbf{Q}_{i}^{-1} \mathbf{H}_{i i} \mathbf{V}_{i},
$$

where $\mu$ is a real constant. With this choice, it is easy to see that the mutual information is changed to (6), which is the capacity obtained by optimum receiver processing. Therefore the chosen MMSE receiver weight vector is optimum.

$$
\mathcal{I}=\log _{2} \operatorname{det}\left[\mathbf{H}_{i i}^{H} \mathbf{Q}_{i}^{-1} \mathbf{H}_{i i} \mathbf{V}_{i} \Phi_{i} \mathbf{V}_{i}{ }^{H}+I_{N_{t}}\right] \text {. }
$$

The eigenvalue decomposition of the matrix $\mathbf{H}_{i i}^{H} \mathbf{Q}_{i}^{-1} \mathbf{H}_{i i}$ is $\mathbf{U} \boldsymbol{\Lambda} \mathbf{U}^{H}$, where $\mathbf{U}$ is an unitary matrix, and $\boldsymbol{\Lambda}$ is diagonal. Note that the number of eigen-modes of the system is determined by the rank of this matrix. With this choice, the mutual information becomes

$$
\begin{aligned}
\mathcal{I}_{i} & =\log _{2}\left|\mathbf{U} \boldsymbol{\Lambda} \mathbf{U}^{H} \mathbf{V}_{i} \Phi_{i} \mathbf{V}_{i}{ }^{H}+I_{N_{t}}\right| \\
& =\log _{2}\left|\boldsymbol{\Lambda}^{1 / 2} \mathbf{U}^{H} \mathbf{V}_{i} \Phi_{i} \mathbf{V}_{i}^{H} \mathbf{U} \boldsymbol{\Lambda}^{1 / 2}+I_{N_{t}}\right|
\end{aligned}
$$

Using Hadamard inequality $\left(|A| \leq \prod_{i} a_{i i}\right)$, the mutual information, $\mathcal{I}_{i}$ is maximized if $\mathbf{U}^{H} \mathbf{V}_{i} \Phi_{i} \mathbf{V}_{i}^{H} \mathbf{U}=$ $\tilde{\mathbf{P}}_{i}$ is diagonal with non-negative elements $p_{i 1}, p_{i 2}, \ldots p_{i N_{t}}$. In this case

$$
\mathcal{I}_{i}=\log _{2} \operatorname{det}\left[\tilde{\mathbf{P}}_{i} \boldsymbol{\Lambda}+I_{N_{t}}\right]=\sum_{j} \log _{2}\left(1+p_{i j} \lambda_{j}\right),
$$

where $\lambda_{j}$ 's are the diagonal elements of $\Lambda$. Since $\mathbf{U}$ is unitary, and $\operatorname{tr}(A B)=\operatorname{tr}(B A)$, then $\operatorname{tr}\left(\mathbf{V}_{i} \Phi_{i} \mathbf{V}_{i}^{H}\right)=$ $\operatorname{tr}\left(\tilde{\mathbf{P}}_{i}\right)$.

\subsection{Constant Power per Subcarrier}

If we assume that the total power of eigen-modes at each subcarrier is fixed, our problem becomes the maximization of $\mathcal{I}_{i}$, with the constraint $\operatorname{tr}\left(\tilde{\mathbf{P}}_{i}\right) \leq P_{i}^{k}$. The answer is the well-known water-filling solution [7], which says

$$
p_{i j}=\left(\nu-\frac{1}{\lambda_{j}}\right)^{+},
$$

where $\nu$ is chosen to guarantee $\sum_{j} p_{i j}=P_{i}^{k}$, and $(x)^{+}=\max (x, 0)$. This equation is used for nonzero $\lambda_{j}$ 's. If $\lambda_{j}=0$, we take $p_{i j}=0$.

To find the optimum transmit weight vectors, we need to solve $\mathbf{U}^{H} \mathbf{V}_{i} \Phi_{i} \mathbf{V}_{i}^{H} \mathbf{U}=\tilde{\mathbf{P}}_{i}$ or

$$
\left(\mathbf{V}_{i} \Phi_{i}^{1 / 2}\right)\left(\mathbf{V}_{i} \Phi_{i}^{1 / 2}\right)^{H}=\mathbf{U} \tilde{\mathbf{P}}_{i} \mathbf{U}^{H} \text { for } \mathbf{V}_{i} \text {. If } N_{t}>T,
$$

then $\mathbf{V}_{i}=\mathbf{U} \underbrace{\left[\begin{array}{c}\mathbf{P}_{1}{ }^{1 / 2} \\ \mathbf{0}\end{array}\right]}_{\mathbf{B}} \Phi_{i}^{-1 / 2}$, where $\mathbf{P}_{1}$ is an $T \times T$

diagonal matrix with nonzero diagonal elements of $\tilde{\mathbf{P}}_{i}$ on its main diagonal. If $N_{t}=T$, then $\mathbf{V}_{i}=\mathbf{U B} \Phi_{i}^{-1 / 2}$, with $\mathbf{B}=\tilde{\mathbf{P}}_{i}^{1 / 2}$. In all cases, $\mathbf{B B}^{H}=\tilde{\mathbf{P}}_{i}$. With these choices, we have

$$
\begin{aligned}
\mathbf{x}_{i}^{k} & =\mathbf{W}_{i}^{k^{H}}(\mathbf{H}_{i i}^{k} \mathbf{V}_{i}^{k} \mathbf{s}_{i}^{k}+\underbrace{\sum_{m \neq i} \mathbf{H}_{m i}^{k} \mathbf{V}_{m}^{k} \mathbf{s}_{m}^{k}+\mathbf{n}_{i}^{k}}_{q}) \\
& =\mu^{*} \Sigma \Phi_{i}^{-1} s_{i}^{k}+\mu^{*} \Phi_{i}^{-1 / 2} \mathbf{B}^{H} \mathbf{U}^{H} \mathbf{H}_{i i}^{k}{ }^{H} \mathbf{Q}^{-1} q
\end{aligned}
$$


where $\Sigma=\mathbf{B}^{H} \mathbf{\Lambda} \mathbf{B}$ is a diagonal matrix, for all 3 abovementioned cases. This result shows that at the receiver, and at each subcarrier, the multiple streams are orthogonal to each other. The receiver covariance matrix for $N_{t}=T$ is

$$
E\left[\mathbf{x}_{i}^{k} \mathbf{x}_{i}^{k H}\right]=\mu^{2} \tilde{\mathbf{P}}_{i}^{2} \boldsymbol{\Lambda}^{2} \Phi_{i}^{-1}+\mu^{2} \tilde{\mathbf{P}}_{i} \boldsymbol{\Lambda} \Phi_{i}^{-1} .
$$

So the SNR for the $j^{t h}$ stream is $p_{i j} \lambda_{j}$. Similar results can be found for other cases.

\subsection{Constant power per user}

In reality the total transmit power for each base station is limited. As a practical limitation, here we assume that total power allocated to each user is fixed. the overall mutual information between the $i^{t h}$ receiver, and its corresponding transmitter, can be found from $\mathcal{I}_{i}=\sum_{k=1}^{K} \mathcal{I}_{i}^{k}$.

If $\tilde{\mathbf{P}}_{i}^{k}=\mathbf{U}_{i}^{k}{ }^{H} \mathbf{V}_{i}^{k} \Phi_{i}^{k} \mathbf{V}_{i}^{k}{ }^{H} \mathbf{U}_{i}^{k}$ for each $k$ is diagonal, by Hadamard inequality, each term of the above sum is maximized regardless of any power constraint. Since each term is positive, the sum will also be maximized. Therefore, we want to maximize

$$
\mathcal{I}_{i}=\sum_{r=1}^{K T} \log _{2}\left(1+p_{i r} \lambda_{i r}\right),
$$

constrained to $\sum_{r=1}^{K T} p_{i r}=P_{i}$, where $P_{i}$ is the power allocated to user $i$. The index $r$ is used to represent all streams and all subcarriers. For a given $r$, the index of the stream is found from $j=(r \bmod T)+1$, and the index of subcarrier from $k=\lfloor(r / T)\rfloor$. Again, The answer is the water-filling solution presented in (8). The SNRs are again $p_{i r} \lambda_{i r}$ at corresponding subcarriers and streams.

\subsection{Iterative Waterfilling}

Note that each transmitter optimizes its transmit spectrum independently of the other transmitters but knowing the interference covariance matrix at its receiver. Since each transmitter is repeating the same process, the interference at the receiver is going to be changed and the above steps should be repeated until the transmit and interference covariance matrices converge. Therefore, the iterative water-filling [4] is used to find the powers at each eigen-mode at each subcarrier of each transmitter. Note that the authors in [4] have used the iterative water-filling for a single-cell single-carrier system, while we are proposing this scheme for multi-cell multicarrier multiple antenna systems.

For the first scheme, in which the constant power per subcarrier is considered, the iterative water-filling at subcarrier $k$ is proposed as follows, starting from the receiver $n=1$ :

Algorithm I [Constant power per subcarrier]

1. Find the interference from Eq. (2).

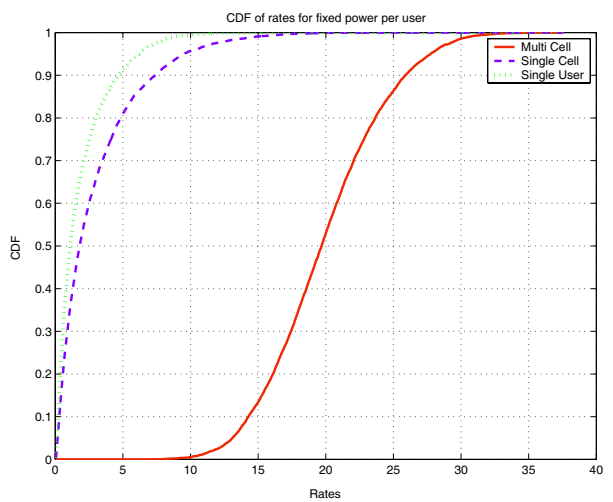

Fig. 1: Achievable rate CDF, for fixed power per carrier

2. Find the eigenvalue decomposition of

$$
\mathbf{H}_{n n}^{k}{ }^{H} \mathbf{Q}_{n}^{k-1} \mathbf{H}_{n n}^{k}=\mathbf{U}_{n}^{k} \boldsymbol{\Lambda}_{n}^{k} \mathbf{U}_{n}^{k}{ }^{H} \text {. }
$$

3. Find the power of each eigen-mode with nonzero eigenvalue from (8) For $\lambda_{j}=0$, take $p_{i j}=0$. Create a diagonal matrix $\tilde{\mathbf{P}}_{n}^{k}$, whose diagonal elements are $p_{i j}$.

4. Find the transmit weight vectors $\mathbf{V}_{n}^{k}=\mathbf{U}_{n}^{k} \mathbf{B} \Phi_{n}^{k-1 / 2}$, when $\mathbf{B}$ is $\tilde{\mathbf{P}}_{n}^{1 / 2}$ for $N_{t}=T$. For the other cases $B$ is found correspondingly.

5. Set $n=n+1$ and continue from step 1 until convergence.

For the second scheme, where the constant power per user is considered, the iterative water-filling is similar to Algorithm $I$, where step 3 is described as follows:

Step 3. for Algorithm II: For $r=1 \ldots K T$, find the power of each eigen-mode with nonzero eigenvalue from (8), For $\lambda_{i r}=0$, take $p_{i r}=0$. For each subcarrier, create a diagonal matrix $\tilde{\mathbf{P}}_{n}^{k}$, whose diagonal elements are corresponding $p_{i r}$ 's.

For each iterative water-filling scheme, the choice of receive weight vectors at a receiver has no effect on the amount of interference at other users. Therefore, the receive weight vectors are evaluated using Eq. (5), after the iterative algorithms are converged.

We have shown that the above iteration will converge to a fixed point starting from any initial transmit and receiver processing matrices.

\section{SIMULATION}

We simulate a wireless network consisting of 100 and 125 co-channel base stations placed in a hexagonal pattern. We assume one or two mobiles randomly distributed in a cell according to a uniform distribution. We use an OFDM system with 32 subchannels for transmission. The communication channel is assumed to follow the COST207 Typical Urban 6-ray channel model. Each 


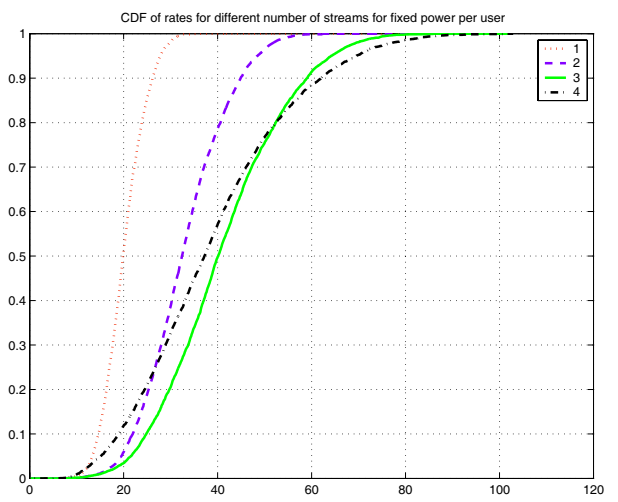

Fig. 2: Achievable rate CDF, for fixed power per user

OFDM symbol is assumed to be $1 \mu \mathrm{s}$ long which corresponds to a bandwidth of $1 \mathrm{MHz}$. The white Gaussian thermal noise power at each receiver is calculated based on a noise figure of $3 \mathrm{~dB}$ and $1 \mathrm{MHz}$ bandwidth. We consider 4 transmit antennas and 4 receive antennas. Independent multiple data streams are transmitted from each base stations to each mobile in its corresponding cell. Two different cases are considered.

Algorithms 2 have been simulated for multi-cell system. Fig. 1 shows the CDF of the achievable rate for 125 cells with of reuse 3 , one mobile per cell, and two transmitted streams. In this figure, we have shown the case where the in-cell interference plus noise is used as an approximation for noise covariance to calculate the transmit weights. It can be seen that when the multicell interference is taken into account, the overall rate is higher. The same CDF for different number of streams per subcarrier is shown in Figs. 2 and 3 for 100 cells with one and two mobiles per cell, respectively. In the first case, as we increase the number of streams, more bandwidth can be assigned per user and the achievable rate per user is increased. However, as we increase the number of co-channel mobiles per cell, more processing is needed to combat the effect of in-cell interferences, and therefore the performance of two streams is better than that of four streams.

\section{CONCLUSION}

We have proposed iterative water-filling solutions for multi-user multi-cell wireless systems where multiple antennas are deployed at both transmitters and receivers. The proposed algorithm assigns multiple independent substreams for each user to increase data rate for each user and is performed as a distributed scheme. That is, each transmitter has only the knowledge of interference at its own receiver and the channel response in that link. We have shown that the algorithm converges to a fixed point solution where the data rate for each user is lo-

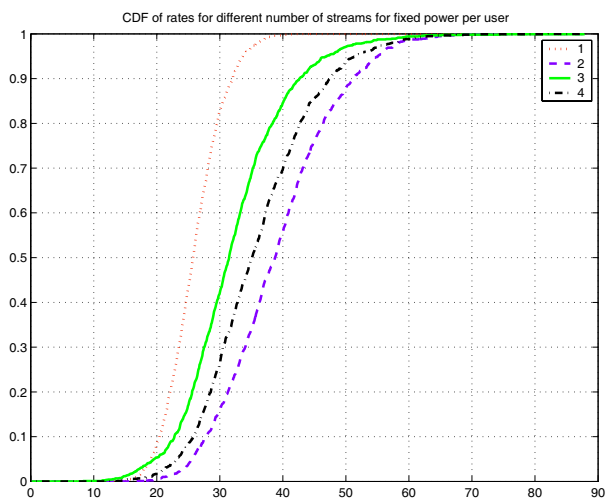

Fig. 3: Achievable rate CDF, for fixed power per user

cally optimized. We have evaluated the performance of the proposed scheme through numerical analysis.

\section{REFERENCES}

[1] G. J. Foschini, "Layered Space-Time Architecture for Wireless Communication in a Fading Environment When using Multi-element Antennas," Bell Labs Tech Journal, pp. 41-59, Fall 1996.

[2] F. Farrokhi, A. Lozano, G. Foschini, and R. A. Valenzuela, "Spectral Efficiency of FDMA/TDMA Wireless Systems with Transmit and Receive Antenna Arrays," IEEE Trans. on Wireless Comm., vol. 1, Oct 2002.

[3] F. Farrokhi, A. Lozano, G. Foschini, and R. A. Valenzuela, "Very high capacity multiple-acess system using multiple transmit and recieve antennas," Bell Labs Technical Memo, Dec 1999.

[4] W. Yu, W. Rhee, S. Boyd, and J. M. Cioffi, "Iterative Water-Filling for Gaussian Vector Multiple Access Channels," IEEE Intrn. Symp. on Inf. Theory, 2001.

[5] W. Su, Z. Safar, M. Olfat, and K. J. R. Liu, "Obtaining Full Diversity Space Frequency Codes from Space-Time Codes via Mapping," IEEE Trans. on Signal Proc., special issue on Signal Processing for MIMO systems, Dec 2002, Nov 2003.

[6] M. Olfat, F. R. Farrokhi, and K. J. R. Liu, "Power Allocation for OFDM using Adaptive Beamforming over Wireless Networks," submitted to IEEE Trans. on Comm. (under revision), May 2003.

[7] T. M. Cover and J. A. Thomas, Elements of Information Theory. John Wiley, 1991. 